\title{
La escuela como espacio para convertirse en profesor: Experiencias de docentes de Educación Física en formación The school as a space to become a teacher: Experiences of Physical Education teachers in training
}

*Samuel Pérez Norambuena, *Raquel Aburto Godoy, **Felipe Poblete-Valderrama, ***Oscar Aguayo Álvarez *Universidad del Bio-bío (Chile), **Universidad Católica de la Santísima Concepción (Chile), ***Universidad San Sebastián (Chile)

Resumen: En la formación de profesores, los futuros maestros aprenden una amplia gama de conocimientos y habilidades. Se ha indicado que la práctica docente es la parte más importante de la formación ya que afecta el desarrollo personal y profesional de los estudiantes de pedagogía. Esta investigación se centró en analizar los significados de las experiencias vivenciadas de 36 docentes en formación, durante un período de práctica docente en escuelas secundarias de la región del BíoBío, Chile. Estudio de tipo cualitativo cuyo instrumento de recogida de información fue la bitácora de registro digital. Los principales hallazgos dan cuenta que los estudiantes docentes experimentan una amplia variedad de emociones, durante este proceso formativo, influenciado por las interacciones que ocurren en el aula, la relación que se produce con los estudiantes, la comunicación con profesores que les guían en la escuela y por el contexto de éstas. Estos elementos interpelan y predisponen su desempeño docente. Los resultados además sugieren que es necesario incorporar, de manera intencionada en la formación, los temas relacionado con las interacciones humanas, ya que son fundamentales para asumir los desafíos futuros como profesores.

Palabras clave: práctica pedagógica; formación de docentes; docencia; formación profesional.

\begin{abstract}
In teacher training, future teachers learn a wide range of knowledge and skills. It has been indicated that teaching practice is the most important part of training since it affects the personal and professional development of pedagogy students. This research focused on analyzing the meanings of the experiences of 36 teachers in training, during a period of teaching practice in secondary schools in the Bío-Bío region, Chile. Qualitative study whose information collection instrument was the digital registry log. The main findings show that student teachers experience a wide variety of emotions during this training process, influenced by the interactions that occur in the classroom, the relationship that occurs with students, communication with teachers who guide them in school and by the context of these. These elements challenge and predispose their teaching performance. The results also suggest that it is necessary to intentionally incorporate issues related to human interactions in training, as they are essential to take on future challenges as teachers
\end{abstract}

Keywords: pedagogical practice; teacher training; teaching; vocational training.

\section{Introducción}

En los procesos formativos de profesores, la formación práctica es un proceso de carácter integral (Barrera \& Hinojosa, 2017). Se da en la relación con los alumnos; la comunidad escolar; el currículo; relaciones interpersonales entre otros. Permiten promover en los estudiantes implicarse con una comunidad escolar y con los distintos actores educativos de manera directa y presencial. La práctica docente ha sido considerada como uno de los elementos claves en los procesos de la Formación Inicial Docente (en adelante FID) y es una oportunidad para que los profesores en formación vivencien

Fecha recepción: 10-03-21. Fecha de aceptación: 08-06-21

Felipe Poblete-Valderrama

felipepobletev@gmail.com algunos de los roles profesionales y exploren, gradualmente, lo que significa ser un maestro en la práctica real y situada (Suckel, Campos, Sáez \& Rodríguez., 2017; Meyer, 2009), permiten consolidar aprendizajes relevantes para su desarrollo profesional (Egido \& López, 2016) y son un espacio que permiten construir la identidad profesional a través de las experiencias positivas y negativas que viven en la escuela (Teng, 2017) .

Dichas experiencias prácticas, para que sean de calidad y contribuyan significativamente en dicha identidad, deben asumir principalmente tres grandes principios: a) Continuidad, es necesario que se presente de manera gradual y creciente complejidad teniendo en cuenta las características del sujeto que aprende, b) Interacción, se plantea la necesidad de entender las situaciones prácticas como ejemplos de la realidad que presenta diversos componentes conceptuales o 
metodológicos, y c) Reflexión, en donde el estudiante en formación analiza lo que hace, los motivos para hacerlo y es capaz de tomar conciencia y darse cuenta de que el trabajo profesional es complejo y multifactorial, y de esta manera la reflexión sobre la experiencia permite el aprendizaje de nuevos conocimientos y saberes (Solís, Núñez, Contreras, Rittershanssen, Montecinos \& Walker, 2011).

En ellas, intentan desplegar las habilidades aprendidas a lo largo de su vida y en el transcurso de su formación universitaria, permitiendo demostrar y responder a los desafíos, propios, de este proceso. Estas instancias, tienen una serie de beneficios para los futuros profesores como el desarrollo de competencias asociadas al proceso de aprender a enseñar (Mansvelder-Longayroux, Beijaard \& Verloop, 2007), de aprender y aplicar habilidades interpersonales (Serrate, Casillas \& González, 2015) las cuales son fundamentales tanto para su desempeño futuro, como para los aprendizajes de sus futuros estudiantes. Esta formación debe centrarse en la práctica de los profesores y en los conocimientos de lo que hacen diariamente; en una formación para reflexionar, evaluar y evaluarse, y aprender, de su práctica, para mejorar continuamente. Además, con conocimientos éticos de la práctica: enseñar para respetar, valorar, convivir e intervenir en el mundo (Villalón, 2016).

(Contreras, Rittershanssen, Montecinos, Solís, Nuñez \& Walker., 2010), indican que existen cinco formas de entender la práctica de los futuros docentes que se caracteriza por ser un espacio: de formación en la universidad en la que se construyen propuestas con base en teorías, la cual ha sido ampliamente abordado por los centros de formación; que tiene en cuenta lo que está sucediendo en la escuela, lo que es procesada en y por la universidad con el fin de interpretar lo que está sucediendo en la realidad escolar; de recolección de información relevante que les permitirá planificar y ejecutar acciones, contextualizadas, en la escuela; de intervención para vivenciar y experimentar desempeños docentes y analizarlos en la universidad con el fin de adquirir y desarrollar competencias, y finalmente como el lugar para diseñar y conducir los procesos de enseñanza y aprendizaje de manera autónoma por parte del docente en práctica.

Además de estas caracterizaciones nos encontramos con algunos enfoques teóricos y conceptos que dan cuenta de los fundamentos que han estado presentes en la formación práctica en la formación de maestros y que permiten una comprensión de ésta:

a) Enfoque conductista- aplicacionista o técnico ins- trumental es una actividad genérica de transmisión de la información, en la cual el practicante asume un rol técnico, pasivo y como un mero ejecutor, cuya finalidad es implementar medidas o reproducir modelos de enseñanza diseñados por expertos (Hirmas, 2014). Aquí existe una separación entre teoría y práctica, que pregona que la fundamentación teórica precede a la práctica. El futuro docente, bajo esta mirada, se presenta como un sujeto que es capaz de transmitir conocimientos, aceptados, relacionados con la teoría que se enseña en las universidades en los procesos de la FID.

b) Enfoque crítico - reflexivo, el cual reconoce la multidimensionalidad, complejidad de los fenómenos educativos y releva el conocimiento situado, que es imprescindible para manejar situaciones de la clase (Darling-Hammond, 2005). Se realiza un análisis crítico de la realidad educativa, en contextos de colaboración y reflexión conjunta, de modo de avanzar hacia una reconstrucción de la conceptualización teórico-práctica (Guerra \& Montenegro, 2017). El practicante, desde este enfoque, asume un rol activo a partir de sus experiencias. «Se concibe a los profesores como generadores de conocimiento al reflexionar sistemática y colectivamente acerca de su quehacer docente» (Hirmas, 2014 , p. 2). La práctica permite realizar un análisis crítico de la enseñanza desde la perspectiva del estudiante y no desde lo promovido en su formación, lo que generaría prácticas pedagógicas adecuadas y pertinentes.

En Chile, existe el consenso respecto a la presencia principalmente del enfoque conductista aplicacionista en los procesos formativos (Guerra \& Montenegro, 2017), en el cual predominan la mirada dicotómica de teoría y práctica la que se presentan de manera separadas en dos momentos diferentes y absolutamente desconectados entre sí (Labra, 2011; Hirmas, 2014; Russell, 2014; Rivas, Cortés \& Márquez, 2018). Predominando en la FID lo teórico disciplinar y relegando, muchas veces, la formación práctica a espacios de menor importancia. Esto ha producido que los procesos de formación no respondan a los desafíos de las escuelas y sus estudiantes, por una parte, y por otra, a las necesidades de los profesores en formación en términos de tener una formación integrada y no parcializada.

Nuestro objeto de estudio se focaliza en la formación práctica de futuros profesores, en la reflexión y el relato de esa experiencia, en algunos aspectos relevantes de su formación práctica, lo que permite abrir nuevos sentidos y posibilidades para y en la FID. Quisimos volcar el foco en los estudiantes y sus reflexiones sobre la propia práctica porque es uno de los puntos débiles 
del proceso formativo que requiere de atención y preocupación (Yangin \& Gungor, 2018). Además, ya que como profesores que formamos docentes, comprendemos la importancia de la formación práctica y la necesidad de escuchar a los estudiantes de pedagogía porque son ellos los que asumirán el relevo, pronto, en la escuela y quienes comprenden de mejor manera los desafíos que requiere su labor futura y los alcances formativos que necesitan ser abordados desde los espacios de la FID.

(Cortez, González \& Padua, 2020), indican que existen distintos focos investigativos en relación a este objeto de estudio y que en lo referido a los practicantes existen tres ejes temáticos en lo que es posible agrupar la voz de los estudiantes sobre la experiencia en la práctica en la escuela: 1) análisis de competencias y tareas el cual básicamente tienen que ver con el desempeño y la reflexión que realizan los estudiantes de la práctica en la que observan críticamente la realidad educativa; 2) propuestas didácticas para el aprendizaje, aquí se investiga la presencia de aspectos didácticos, en la práctica, que favorecen los aprendizajes como blogs, creación de diarios u otras estrategias; 3) indagaciones sobre la identidad y sentido otorgado a la práctica. Éste último eje tiene relación con como la práctica y la FID favorecen y promueven la identidad profesional en docentes.

Este artículo tiene como objetivo develar las experiencias y reflexiones sobre la práctica, de profesores en formación de la Carrera de Pedagogía en Educación Física, lo que permite conocer cómo experimentaron y percibieron su desempeño en los distintos establecimientos educativos asignados. La importancia del estudio de las experiencias y reflexiones de los futuros profesores es que producen un espacio de incertidumbre entre lo que hicieron, lo que hacen, lo que podría hacer y las consecuencias de la acción (Álvarez \& Hevia, 2013; Solís et al., 2011).

\section{Material y Método}

El estudio fue realizado y se sustenta en los principios del paradigma interpretativo, con un diseño cualitativo basada en el análisis y la interpretación del blog digital narrados por los estudiantes participantes. El cual nos brindó la posibilidad de aproximarnos a las relaciones presentes entre la información expresada, por los participantes, y el contexto donde se obtuvo, permitiendo de esta manera la comprensión de las significaciones producidas (Guba \& Lincon, 2002). Se utilizó el enfoque fenomenológico ya que este permite el estudio de la experiencia, del mundo de la vida y de la cotidianidad de estos actores educativos a partir de sus narraciones en un diario virtual.

\section{Sujetos de estudio}

Los participantes del estudio fueron 36 estudiantes que cursaban la asignatura práctica intermedia, de la Carrera de Pedagogía en Educación Física de una Universidad del sur de Chile (mujeres: 12, hombres: 24; promedio de edad: 22 años). Esta es una asignatura de la formación práctica, del sexto semestre. Es la primera experiencia que los enfrenta a un establecimiento educacional en el nivel de enseñanza media, y es desarrollada por las y los estudiantes de manera individual durante aproximadamente ocho semanas, en las cuales en una primera etapa deben conocer el establecimiento, su proyecto educativo, el curso que van a intervenir, los ejes curriculares y objetivos que trabajarán posteriormente. Además, en este proceso observan las clases del profesor guía y su desempeño. Posteriormente deben planificar, seis sesiones, una unidad de aprendizaje y plantear junto a su profesor una evaluación de los contenidos tratados.

Los estudiantes participaron de manera voluntaria y bajo un consentimiento informado dieron a conocer su interés en participar de este estudio. Se dejaron fuera del análisis algunas bitácoras por no tener la información en su totalidad. De acuerdo con los objetivos perseguidos, la muestra tuvo los siguientes criterios de elección: estudiantes del sexto semestre de la carrera; que estuvieran cursando la práctica de tercer año con cursos de enseñanza media; que hayan realizado seis narraciones de la experiencia del proceso como profesor practicante, en un blog digital y que esas narraciones hayan sido expresadas reflexivamente.

\section{Instrumento}

El instrumento aplicado fue el blog digital los cuales fueron construidos y creados por ellos en el que debían organizar sus ideas sobre lo trabajado clase a clase y en el que además se les solicito reflexionar principalmente a partir de los incidentes críticos que estaban viviendo, lo que han sido definido como «aquellos sucesos cotidianos, extraídos de la propia experiencia, que sorprenden por su buen o mal curso e inducen a reflexión» (Padilla y Costa, 2017, p. 61).

Los blog han sido considerados como una herramienta que estimula el pensamiento crítico y reflexivo, el intercambio de ideas en los estudiantes (Úbeda \& Molina, 2016). Dicho espacio digital nos permitió recolectar 
información concerniente a los procesos mentales por los cuales atravesaban estos estudiantes en el proceso que les toco enfrentar. En el cual debieron comunicar y expresar de manera escrita sus autoreflexiones sobre esta experiencia formativa.

La autoreflexión, que era solicitada y que debían narrar y escribir en su diario posteriormente a la realización de sus intervenciones como practicante, ha sido valorada por diferentes estudios como un atributo crítico y necesario para la enseñanza y el aprendizaje efectivo (Beauchamp \& Thomas, 2009; Rich \& Hannafin, 2009).

\section{Procedimiento de recogida y análisis de datos}

Para el análisis fenomenológico se analizaron un total de 216 relatos ingresados en su blog por los estudiantes sobre sus vivencias y devenires en el proceso de práctica. Los cuales fueron analizados a partir de la propuesta de (López, 2010) para lo cual primeramente se redujo la información recopilada, posteriormente se codificaron los registros comunicativos extraídos de cada blog, permitiendo acercarnos a la esencia de los significados compartidos intersubjetivamente, por estos profesores en formación, sobre la experiencia vivida y relatada en relación a la práctica pedagógica. De esta manera fueron divididos los textos en unidades de significado, englobadas en diferentes categorías emergentes, a partir de la concentración de significados.

El comienzo de las categorías inductivas se generó a partir de la identificación de los conceptos claves mediante la frecuencia de palabras expresadas, por los profesores en formación, en sus narrativas.

Este estudio contó con el visto bueno del Comité Asesor de Bioética y Bioseguridad de Investigación de la Universidad del Bío-Bío.

\section{Resultados}

A continuación, se muestran los análisis de los resultados, a partir de una primera aproximación cuantitativa, a las categorías centrales, que da cuenta de la presencia porcentual de temáticas aparecidas en el análisis de las bitácoras, la que posteriormente nos posibilito realizar el análisis cualitativo de las experiencias narradas por estos estudiantes de pedagogía en Educación Física. En la figura $\mathrm{N}^{\circ} 1$ Se observa la importancia otorgada por los estudiantes a los temas emocionales, en el cual más de un $30 \%$ de los conceptos pesquisados se relacionan con este tema y se encuentra a 8 puntos porcentuales sobre las interacciones que se dan en el aula que es el segundo concepto que más presencia tienen en las narraciones. En ellos aparece mencionado el miedo a una situación y relaciones nuevas, y la alegría frente a lo que sucede con sus desempeños. Es decir, ellos vivenciaron y experimentaron los desafíos emocionales que conlleva el trabajo docente. Estos resultados dan cuenta de la necesidad de abordar esta dimensión en los procesos formativos dichas temáticas.

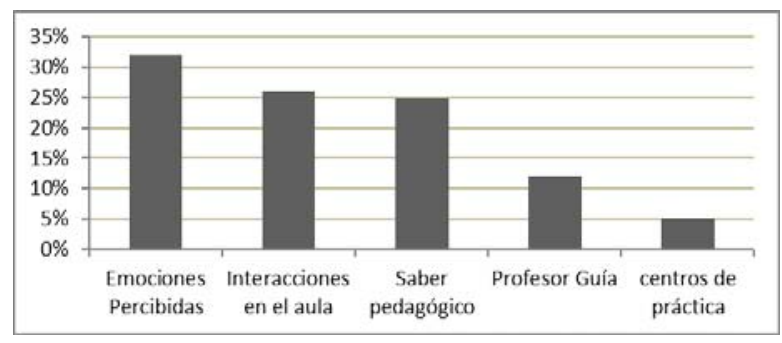

Figura 1. Presencia porcentual de temáticas aparecidas en el análisis de las bitácoras.

Hubo, además una alta cantidad de temas relacionadas con el saber pedagógico, necesario y pertinente que se debe implementar en este proceso. Podemos desprender que es un objeto de importancia para los practicantes debido a que las metodologías y técnicas utilizadas les permiten desarrollar adecuadamente los objetivos de aprendizaje y los contenidos disciplinares planificados. Los cuales deben ser variadas y llamativas para que los estudiantes de la escuela participen activamente. Un poco más lejano aparece la categoría que hace referencia al profesor guía, quien tiene un grado importante de influencia en los practicantes, ya que en sus clases de observación obtienen una impresión del profesor guía que puede servirles como un modelo a seguir o no. Por último, respecto de la categoría centros de práctica, no se describen tantas experiencias, lo que da a entender que el contexto donde desarrollan sus prácticas no es tan importante y relevante como las otras variables, para estos estudiantes de pedagogía.

A continuación, realizaremos un análisis descriptivo de las categorías centrales aparecidas en las narraciones de los estudiantes participantes. De manera esquemática en la tabla $\mathrm{N}^{\circ} 1$ : Categorías sobre la experiencia en la práctica, se pueden apreciar estas categorías que emergieron de sus relatos: Emociones percibidas, Interacciones en el aula, Saber pedagógico, Profesor guía y Centros de práctica las que representan el significado de las experiencias de los estudiantes sobre la práctica pedagógica.

\begin{tabular}{|c|c|}
\hline Categoría & Concentración de significados \\
\hline Emociones percibidas & Expectativas; motivación \\
\hline Interacciones en el aula & Disciplina; respeto \\
\hline Saber Pedagógico & Aplicación de la didáctica \\
\hline Profesor Guía & Didáctica; relación \\
\hline Centros de práctica & Infraestructura; contexto social. \\
\hline
\end{tabular}




\section{Emociones Percibidas}

La concentración de significados se relacionó con las emociones percibidas que fueron expresadas a través de sensaciones, percepciones y sentimientos negativos o positivos, «en reiteradas ocasiones había que tomarles la atención de una u otra manera, pero nada parecía servir y me angustiaba que como pasaban los días y se acercaba la hora en que yo tendría que estar en frente pidiendo que se comportaran» (H.L.). Este relato da cuenta de cómo los factores emocionales influyen en la percepción de ambientes amenazantes o seguros y provocando e incidiendo en la disposición y desempeños con que deben realizar su práctica. A este hecho GómezTorres (2015) lo definió dimensión relacional en la que los factores contextuales son determinantes para provocar determinadas emociones

Las emociones a partir de lo contextual les predisponen a actuaciones que les provocan desajustes «desde la noche anterior a mi primer día de práctica ya tenía un poco de nerviosismo, traté de dormir temprano para tener todas las energías posibles al día siguiente.... los nervios se pronunciaron más, el desayuno me dejó satisfecho más rápido». (B.V), y y también les promueven sensaciones de bienestar «si bien me encontraba muy cansada también estaba motivada por ver la alegría de algunos y cansancio de otros, finaliza mi clase y recibo el afecto de los niños que mejor regalo» (P.J.)

Estas narraciones sobre la dimensión emocional concuerdan con la importancia de los aspectos emocionales, debido a su rol fundamental en los procesos de enseñanza y aprendizaje Hargreaves (2003).

Es relevante comprender la importancia de este tema en los futuros docentes, ya que las emociones que se experimentan cuando se está aprendiendo a convertirse en profesores probablemente reflejen no solo su aprendizaje durante la FID, sino también sus experiencias, futuras, como docentes activos en el sistema educativo (Rowe et al., 2015). Lo cual nos indica que esta dimensión de la experiencia sea un factor preponderante en la identidad profesional de los futuros profesores.

«...Mi primera clase fue un desastre, la mayoría de los alumnos no tenía interés, sobre todo las niñas, no tenía el apoyo de nadie y me sentí sobrepasado». (J. Z).

La cita anterior no muestra como la formación docente también puede ser vivida como un momento de quiebre y angustia, lo que (Goetz et al., 2014) denomino como un entorno de aprendizaje perjudicial y estresante. Por ello la necesidad de abordar los temas emocionales en los procesos formativos, de manera intencionada y sistemática ya que no hacerlo puede gene- rar estados de preocupación, desgaste y desmotivación en los futuros profesores y retrasar la consolidación de la identidad profesional.

\section{Interacciones en el Aula}

Esta categoría hace referencia al ambiente para el aprendizaje, que se genera en la sala de clases, el cual influye en las dinámicas escolares. Está compuesto principalmente por las interacciones entre los alumnos del curso en el que se desempeña el practicante, los cuales en ocasiones interfieren, condicionan su experiencia y disposición para desarrollarla exitosamente. En ocasiones las interacciones les provocan desgaste como en este caso les produce ciertos grados de incertidumbre «...Se desordenan mucho, me cuesta mantener la disciplina y lograr que trabajen, cada clase hay que insistir bastante para que se muevan un poco». (C. E) y en otras inamovilidades «los alumnos comenzaron a tirarse papeles dos alumnas estaban con el celular. Ahora en parte entiendo a la directora que decía que eran desordenados y no querían hacer nada, porque realmente era así». Sacristán (2008), al respecto señala que los estudiantes en práctica no muestran cultura de enfrentamiento de los conflictos; al contrario, consideran válida la idea de intentar ser indiferentes al hecho, ignorando lo sucedido y esperando que la situación se resuelva de manera natural «...La relación que tenían entre ellos no era la adecuada, se trataban mal, se gritaban miles de cosas e incluso se trataban con groserías, se molestaban, etc.» (M. S.)

También existen espacios interacciónales que les generan mucha motivación y entusiasmo con el proceso que lideran e invitan a «Esta es mi última semana, creo que echaré de menos al curso, he pasado más tiempo con ellos en orientación que en especialidad y los he aprendido a conocer un poco más. Creo que de alguna u otra forma me fueron aceptando y confiando en mí y eso se agradece» Además, en esos procesos interacciónales los futuros profesores demuestran su vocación y la empatía con la que asumen su labor (Pérez \& Linzmayer, 2018).

El papel de las interacciones y el clima del aula son elementos fundamentales en los procesos de enseñanza y aprendizaje. Se ha señalado que las interacciones entre docente y estudiante y entre estudiantes condicionan los aprendizajes, el desarrollo de habilidades de socialización y de regulación emocional, lo que afecta los índices de deserción escolar y el rendimiento académico (Spaulding et al., 2008). 


\section{Saber Pedagógico}

Este saber tiene relación con la forma en que los practicantes ocupan técnicas y estrategias en el desarrollo de sus clases, en la entrega de contenidos y la forma de evaluación de estos. Estos elementos fueron adquiridos y aprendidos en su formación universitaria y son parte fundamental de su quehacer como futuros profesores de Educación Física. En lo relativo a su función pedagógica, la experiencia, se centró en ámbitos metodológicos y estrategias didácticas, lo que está en consonancia con investigaciones que muestran que los estudiantes de pedagogía tienden a centrar su interés en aprender a desarrollar los aspectos procedimentales del proceso de enseñanza (Montecinos et al., 2012).

«... los alumnos disfrutaron bastante la clase en comparación a las anteriores donde realizaban el salto de espalda, considero que la idea de trabajar en equipo es mucho más motivante que realizar actividades en donde depende de mi propio esfuerzo.» (F. W).

Los alumnos al realizar sus intervenciones cuentan y reflexionan sobre cómo hacen sus clases, la estructura, orden, los procedimientos, entre otros. El método de clase es una herramienta fundamental e importantes en estos procesos de práctica, ya que es lo facilita la entrega de los contenidos a los estudiantes aprendidos, teóricamente, en la Universidad.

«...En la segunda clase continué con el tema, tratando de hacerlo más lúdico para que se integrarán todos y yo siendo más activo de la clase para causar motivación en los niños y niñas.» (J. Z., segunda clase).

\section{Profesor Guía}

Es la persona del establecimiento que acompaña, observa y orienta su desempeño como profesor en formación. Esta relación es muy importante ya que genera o no comodidad y seguridad. Las interacciones con los profesores guías, son importantes para el desarrollo personal y profesional de los profesores practicantes. Esas relaciones influencian de distinta manera en su adaptación, desempeños y satisfacción en la práctica. Esta relación ha sido sindicada como clave para la calidad del proceso de aprendizaje del futuro profesor (Jaspers et al., 2014). Lo que hace necesario, dada su importancia, que los centros formadores e profesores se vinculen de manera efectiva con estos actores.

«... a veces siento que la profesora no me toma en cuenta, debo andar detrás de ella para saber que va a hacer, hoy llegue a la sala de profesores y no me saludo... igual me sentí mal porque ella no me tomaba en cuenta» (C. M.).
Respecto a la didáctica que utilizó el profesor guía, los estudiantes miran sus estrategias y las evalúan de acuerdo con sus creencias y aprendizajes adquiridos en la universidad.

«...Hoy el profesor realizó una clase de basquetbol, según mi punto de vista, fue una clase demasiado técnica para los niños. Por esa misma situación creo yo que los niños no lo tomaban mucho en cuenta y no participaban de la clase». (M. O.).

\section{Centro de práctica}

Unidades educativas donde se desarrollan las prácticas pedagógicas, los cuales tienen una serie de características organizacionales, de infraestructura o implementación las cuales influyen en el desarrollo de su práctica. Las características del centro de prácticas y el contexto social en que se inserta son elementos que condicionaron sus experiencias y las relaciones que se dieron en la práctica. Este elemento concuerda con los hallazgos de (Mendoza \& Covarrubias, 2014; Rodríguez et al., 2017), que indican que los centros educativos son considerados como un promotor de experiencias que promueve la movilización de competencias en los futuros maestros.

«...En general, la escuela no cuenta con muchos materiales y el espacio es reducido para poder realizar la clase». (A. J.).

También esas unidades se insertan en espacios sociales que influyen en su práctica. Los estudiantes apuntan características de sus alumnos que creen importantes, como lugar donde viven.

«...Los estudiantes viven muy cerca de la escuela, por lo que se lo que cuesta vivir ahí, muchos se quedan en la escuela horas después de terminar las clases, y su mundo es la población y su escuela.» (D. G.).

\section{Discusiones}

Los programas de formación docente promueven en los docentes conocimientos teóricos y habilidades prácticas que les permitan responder a los desafíos que deberán enfrentar en los contextos reales de la enseñanza con todo el significado personal y profesional que conlleva esa acción. Lo que demuestra que convertirse en maestro es un itinerario cargado de experiencias y emociones diversas y provocadoras (Kelchtermans \& Deketelaere, 2016). En el cual el vaivén de emociones se hace presente, constantemente en esta parte del proceso formativo, y que permanece presente en la labor docente de tal forma que la labor del profesor involucra 
interacciones complejas a largo plazo y resolución de problemas sociales (Anttila, Pyhältö, Soini, \& Pietarinen, 2016), en este sentido estas experiencias pueden ser transformadas en aprendizajes explícitos (Bächler et al., 2020). Miedo, angustia, alegría y motivación están conviviendo de manera cotidiana en este proceso que debieron asumir. Estos datos concuerdan con lo expresado por (Kim \& Cho, 2014), quienes indicaron que los maestros en formación tienden a experimentar diversos grados de conmoción frente a la realidad escolar. La cual está influida a veces por las ideas previas sobre el rol y las funciones que debe asumir en los periodos de práctica. Por otra parte, nuestros hallazgos se relacionan con los encontrados por (Hinojosa, Hurtado \& Magnere, 2020) quienes resaltan en sus resultados las dificultades que presentan en el ejercicio profesional docente referido en habilidades para resolver problemas emergentes.

Nuestros datos y los resultados de otros estudios, como el de (Bächler, Meza, Mendoza \& Poblete, 2020; Malderez, Hobson, Tracey \& Keer, 2007) nos lleva a sugerir que la formación inicial de profesores debe abordar el componente emocional y el bienestar de los estudiantes. Esto, motivado por las experiencias de los futuros profesores, en las cuales permanentemente se ven expuestos a situaciones que comprometen sus capacidades, sus desempeños y motivaciones. Por lo que se hace relevante crear entornos de aprendizajes adecuados que inviten a activar emociones constructivas, a desarrollar espacios que fomentes las habilidades emocionales que les permitan enfrentar los desafíos actuales $\mathrm{y}$ futuros.

Las principales barreras, para que esta experiencia haya sido ampliamente satisfactoria, con las que se encontraron estos estudiantes se pueden sintetizar en lo siguiente: sus expectativas respecto a su rol y desempeño; los ambientes de aprendizaje en las escuelas, los cuales les provocaban al no poder responder adecuadamente en situaciones de conflicto del aula; la relación con el profesor supervisor en ocasiones fue distante y fría. Esta última es necesario tenerla en cuenta, en los procesos formativos, ya que las relaciones de los estudiantes practicantes con sus profesores supervisores son claves en el equilibrio emocional, en los momentos críticos, de estos futuros profesores (Caires \& Almeida, 2007).

También hablan de sus fortalezas que percibieron en el proceso de autorreflexión entre las que podemos destacar el papel de las relaciones con los estudiantes y profesores supervisores en la experiencia de convertirse en un alumno maestro. Además, el tiempo que ocuparon para enfrentar a estos elementos provocadores, es un buen compañero para ir avanzando en lograr los objetivos personales y académicos. Es decir, los procesos adaptativos, en el caso de estos futuros profesores, se ven altamente influenciados por el tiempo de dedicación y el compromiso con que asumen este desafío.

En base a los resultados y sus implicaciones educativas, hacemos algunas sugerencias en la formación inicial de profesores que permita contribuir a disminuir los grados de incertidumbre y angustia al vincularse con la realidad escolar. En primer lugar, sugerimos que los programas de formación incluyan y declaren intencionadamente y de manera preponderante la adquisición de habilidades emocionales, relacionales y adaptativas. Y que no sean solo asumidas en talleres y cursos complementarios, sino más bien que sea tan importante como las otras áreas de formación del profesorado que han sido el foco esencial en el último tiempo. Por lo tanto, se debe planificar un plan de estudios para que los futuros docentes en formación estén preparados, en lo pedagógico, emocional y psicológico, para asumir los enormes desafíos escolares de manera adecuada. Para esto es fundamental que la percepción del profesorado en formación sea relevada como un indicador valido en si mismo para reorientar las prácticas formativas llevadas a cabo, esto se relaciona con el estudio de (PobleteValderrama, Linzmayer, Matus, Garrido \& Flores, 2018) quienes concluyen que existen diferencias significativas en la valoración de las áreas formativas: pedagógica, educacional, disciplinar y ciencias biológicas. Por otra parte, coincidimos que para la adquisición de habilidades socioemocionales es fundamental que sean consideradas en los procesos de FID en PEF, lo que concuerda con el estudio de (Paez \& Hurtado, 2019) establece que, las instituciones superiores deben asumir la importancia del desarrollo y/o la adquisición de este tipo de competencias.

Sugerimos que la reflexión personal y colectiva sea relevada al lugar que deben tener en los procesos formativos de docentes. Esto permitirá a los futuros profesores mirar los espacios escolares de manera crítica y permitiendo equilibrar expectativas y la preparación recibida. Lo que generará en estos la habilidad para reflexionar sobre su desarrollo profesional, la preparación que tienen, y requieren, para convertirse en los profesores que esperan y buscan ser, lo que concuerda con lo pesquizado por (Alonso, Gómez-Alonso, PérezPueyo \& Gutiérrez-García, 2016) quienes concluyen que las practicas pueden aportar valor a los procesos de formacion del profesorado como formas de exposicion 
gradual a la realidad de la docencia, ayudando a observar errores de intervencion didactica a partir de los cuales es posible generar procesos de analisis y reflexion para la mejora docente.

Para finalizar es posible indicar que la experiencia vivida y relatada no es solamente una experiencia educativa situada en un espacio escolar, en un momento dado de su formación como profesores, en la que desempeñan una labor docente, sino que, además, es una experiencia personal que presenta una fuerte componente social y emocional, la que se ve expresada en la importancia otorgada a elementos personales e interpersonales que deben desplegar y que están interpelándoles frecuentemente más allá de la disciplinar. Es decir, la práctica es un espacio transformador del sujeto por medio de las relaciones interpersonales, las circunstancias físicas y emocionales (Geber \& Nyanjom, 2009), las cuales hoy impactan en estos profesores en formación y en el futuro impactarán a sus estudiantes y las vidas de éstos. Es así como la formación de profesores de Educación Física en Chile debe transitar desde una racionalidad técnica a una más pedagógica para el desarrollo humano como lo plantea (Castillo-Retamal, Almonacid-Fierro, Castillo-Retamal \& Bássoli de Oliveira, 2020).

\section{Conclusión}

Nuestros resultados indican que el proceso de práctica pedagógica es un espacio de interacciones y relaciones sociales que generan cierto grado de incertidumbre emocional permanente en los profesores en formación, el cual es necesario que la FID tenga en cuenta.

La formación inicial de profesores debe tener más en cuenta los estados emocionales y el bienestar de los alumnos. Esto, debido a que estos futuros profesores, constantemente, se ven enfrentados a situaciones que comprometen sus capacidades, sus desempeños y motivaciones

\section{Referencias}

Ahonen, E., Pyhältö, K., Pietarinen, J. \& Soini,T. (2015). Becoming a teacher Student teachers' key learning experiences. International Journal of Higher Education, 4(1), 151-165. https: / /doi.org/10.5430/ijhe.v4n1p151

Alonso, M., Gómez-Alonso, M., Pérez-Pueyo, Á., \& GutiérrezGarcía, C. (2016) Errores en la intervención didáctica de profesores de Educación Física en formación: perspectiva de sus compañeros en sesiones simuladas. Retos 29, $229-235$.

Álvarez, C. \& Hevia, I. (2013). Possibilities and limits of the relationship between theory and practice in initial teacher training. Cultura y Educación, 25(4), 337-346. https:/ / doi.org/ 10.1174/113564013807749759

Anttila, H., Pyhältö, K., Soini, T. \& Pietarinen, J. (2016). How does it feel to become a teacher? Emotions in teacher education. Social Psychology of Education, 19, 451-473. https://doi.org/ 10.1007/s11218-016-9335-0

Bächler, R., Meza, S., Mendoza, L. \& Poblete, O. (2020). Evaluación de la Formación Emocional Inicial Docente en Chile. Revista de estudios y experiencias en educación, 19 (39), 75-106.

Barrera, M. \& Hinojosa, C. (2017). Incidencia del proceso de la práctica profesional en las dimensiones de la formación docente de estudiantes de pedagogía en Educación Física. Revista de Investigación Educacional Latinoamericana, 54 (2), 1-15. https: / /doi.org/10.7764/PEL.54.2.2017.8

Beauchamp, C. \& Thomas, L. (2009). Understanding teacher identity: An overview of issues in the literature and implications for teacher education. Cambridge Journal of Education, 39 (2), 175-189. https://doi.org/10.1080/03057640902902252

Caires, S. \&Almeida, L. (2007). Positive aspects of the teacher training supervision: The student teachers' perspective. European Journal of Psychology of Education, 22(515). https: / / doi.org/10.1007/BF03173469

Castillo-Retamal, F., Almonacid-Fierro, A., Castillo-Retamal, M. \& Bássoli de Olibeira, A. (2020). Formación de profesores de Educación Física en Chile: una mirada histórica. Retos 38, 317- 324.

Contreras, I., Rittershaussen, S., Montecinos, C., Solís, M. C., Núñez, C., \& Walker, H. (2010). Los centros educativos como espacios para aprender a enseñar:Visiones desde los programas de formación de profesores de educación media. Estudios Pedagógicos, 36 (1), 85-105. http://doi.org/10.4067/S071807052010000100004

Cortés, P., González, B. Alba y Padua. D. (2020). Visiones del alumnado y el sentido del prácticum en educación primaria. Revista Interuniversitaria de Formación del Profesorado, 95(34.2),275-298

Darling-Hammond, L. (2005). Educating the New Educator: Teacher Education and the Future of Democracy. The New Educator. 1 (1), 1-18. https://doi.org/10.1080/ 15476880490441379

Egido, I., y López, E. (2016). Condicionantes de la conexión entre la teoría y la práctica en el Prácticum de Magisterio: Algunas evidencias a partir de TEDS-M. Estudios sobre Educación, 30, 217- 237. http://doi.org/10.15581/004.30.217-237

Geber, H., \& Nyanjom, J. (2009). Mentor Development in Higher Education in Botswana: How Important Is Reflective Practice? South African Journal of Higher Education, 23, 5, 894-911. https: / /doi.org/10.4314/sajhe.v23i5.48807

Goetz, T., Frenzel, A. C., Hall, N. C., Nett, U., Pekrun, R., \& Lipnevich, A. A. (2014). Types of boredom: An experience sampling approach. Motivation and Emotion. 38 (3), 401-419. doi:10.1007/s11031-013-9385-y

Gómez-Torres, F. (2015). Identidad y emociones. Praxis Pedagógica, 16, 39-52.

Guba, E., \& Lincoln, Y. (2002). Paradigmas en competencia en la investigación cualitativa. En Denman, C. \& Haro, J.A (comp.), Por los rincones. Antología de métodos cualitativos en la investigación social (p. 113 - 145). Sonora, México: Colegio de Sonora.

Guerra, P. \& Montenegro, H. (2017). Conocimiento pedagógico: explorando nuevas aproximaciones. Educação e Pesquisa. 43 (3), 
663-680. https: //doi.org/10.1590/s15179702201702156031

Hargreaves, A. (2003). Teaching in the Knowledge Society: Education in the Age of Insecurity. Teachers College Press.

Hinojosa, C., Hurtado, M., \& Magnere, P. (2020). Profesores noveles en Educación Física: percepciones sobre su formación docente en base al desempeño del sistema escolar. Retos, 38, 396-405.

Hirmas, C. (2014). Tensiones y desafíos para pensar el cambio en la formación práctica de futuros profesores. Estudios Pedagógicos, 40 (1), 127-143. https://doi.org/10.4067/S071807052014000200008

Jaspers, W., Meijer, P., Prins, F., \& Wubbels, T. (2014). Mentor teachers: Their perceived possibilities and challenges as mentor and teacher. Teaching and Teacher Education, 44, 106-116. https: / /doi.org/10.1016/j.tate.2014.08.005

Kelchtermans, G., \& Deketelaere, A. (2016). The emotional dimension in becoming a teacher. In J. Loughran \& M. L. Hamilton (Eds.), International handbook of teacher education (pp. 429-461). Singapore: Springer.

Kim, H. , \& Cho,Y. (2014). Preservice teachers' motivation, teacher efficacy, and expectation of reality shock. Asia-pacific Journal of Teacher Education, 42 (1), 67-81. https://doi.org/10.1080/ 1359866X.2013.855999

Labra, P. (2011). Construcción de conocimiento profesional docente: el caso de la formación en la práctica [Tesis Postgrado, Universidad Academia de Humanismo Cristiano]. Biblioteca Digital U.A.H.C. http://bibliotecadigital.academia.cl/ handle/123456789/679

López, S. (2010). Las complejidades emergentes en las historias de vida de los «buenos profesores». Polis, 9 (25), 255-267.

Malderez, A., Hobson, A., Tracey, L. \& Kerr, K. (2007) Becoming a student teacher: core features of the experience. European Journal of Teacher Education, 30 (3), 225-248. https: / / doi.org/ 10.1080/02619760701486068

Mansvelder-Longayroux, D., Beijaard, D. \& Verloop, N. (2007). The portfolio as a tool for stimulating reflection by student teachers. Teaching and Teacher Education, 23 (1), 47-62. https: / / doi.org/10.1016/j.tate.2006.04.033

Mendoza, M., \& Covarrubias, C. (2014). Competencias profesionales movilizadas en el prácticum: percepciones del estudiantado del grado de maestro en educación primaria. Revista Actualidades Investigativas en Educación, 14 (3), 1-24. https: //doi.org/ 10.15517/AIE.V14I3.16089

Meyer, D. (2009). Entering the emotional practices of teaching. In P. A. Schutz \& M. Zembylas (Eds.), Advances in teacher emotion research: The Impact on Teachers' Lives (pp. 73-91). Springer Publishing.

Montecinos, C., Walker, H., Cortez, M. \& Maldonado, F. (2012). ¿Por qué y para qué los centros escolares reciben a estudiantes de pedagogía en práctica? Perspectivas de docentes directivos. Páginas de Educación. 6 (1), 37-59.

Padilla, .C y Costa, A. (2017). Análisis de incidentes críticos: una herramienta para aprender de los errores. Revista educación médica. Volumen 19, (1) 60-63 DOI: 10.1016/ j.edumed.2017.06.001

Paes, J. \& Hurtado, J. (2019). Formación inicial docente en profesores de educación física. Levantamiento de competencias específicas a partir de las necesidades del medio educativo. Retos 35, 61 - 66.

Pérez, S. \& Linzmayer, L. (2018). Los buenos profesores en la mirada de padres y apoderados. Educación y Educadores. 21 (3), 373-
387. https:/ / doi.org/10.5294/edu.2018.21.3.1

Poblete-Valderrama, F., Linzmayer Gutiérrez, L., Matus Castillo, Carlos., Garrido Mendez, A., \& Flores Rivera, C. (2018). Percepción de estudiantes de Pedagogía en Educación Física hacia sus profesores. Retos 33, $143-147$.

Rich, P. \& Hannafin, M. (2009). Video annotation tools:Technologies to scaffold, structure, and transform teacher reflection. Journal of Teacher Education, 60(1), 52-68.https: / / doi.org/10.1177/ 0022487108328486

Rivas, J., Cortés, P., y Márquez, M. (2018). Experiencia y contexto: formar para transformar. En C. Monge-López y P. Gómez (coords.). Innovando la docencia desde la formación del profesorado. Propuestas y realidades (pp. 109-124). Madrid: Síntesis.

Rodríguez, D. Armengol, C. \& Meneses, J. (2017). La adquisición de las competencias profesionales a través de las prácticas curriculares de la formación inicial de maestro. Revista de Educación, 376, 229-251. https: / / doi.org/10.4438/1988-592X-RE-2017376-350

Rowe, A. D., Fitness, J. \&Wood, L. N. (2015). University student and lecturer perceptions of positive emotions in learning. International Journal of Qualitative Studies in Education, 28(1), 1-20. https: / /doi.org/10.1080/09518398.2013.847506

Russell,T. (2014). La práctica en la formación de profesores: tensiones y posibilidades en la experiencia de aprender a enseñar. Estudios Pedagógicos, Valdivia, 40, 223-238.

Sacristán, G. (2008): El valor del tiempo en educación. Morata.

Serrate, S., Casillas, S. \& González, M. (2015) Percepción de los estudiantes del Grado en Pedagogía y Educación Social sobre la organización, la utilidad y formación del Practicum. Enseñanza \& Teaching: Revista Interuniversitaria de Didáctica, 33(2): 171 190. https: / / doi.org/10.14201/et2015332171190

Solís, M., Nuñez, C., Contreras, I., Rittershaussen, S., Montecineos, C. \& Walker, H. (2011). Condiciones de la formación práctica de los futuros profesores. Estudios Pedagógicos, 37 (1), 127-147. http: / /doi.org/10.4067/S0718-07052011000100007

Spaulding, S., Irvin, L., Horner, R., May, S., Emeldi, M., Tobin, T. \& Sugai, G. (2008). Schoolwide Social-Behavioral Climate, Student Problem Behavior, and Related Administrative Decisions: Empirical Patterns From 1,510 Schools Nationwide. Journal of Positive Behavior Interventions. 12(2), 69-85. https://doi.org/ 10.1177/1098300708329011

Suckel, M., Campos, D., Sáez, G. \& Rodríguez, G. (2019). Educational trajectories of primary school teachers in training and the construction of pedagogical wisdom. Revista de estudios $\mathrm{y}$ experiencias en educación. 18(36), 117-133. https: / / doi.org/ 10.21703/rexe.20191836suckel14

Teng, F. (2017). Emotional Development and Construction of Teacher Identity: Narrative interactions about the Pre-service Teachers' Prácticum Experiences. Australian Journal of Teacher Education, 42(11), 117-134.

Úbeda, J. \& Molina, J (2016). El blog como herramienta didáctica en Educación Física: la percepción del alumnado. Apunts. Educación Física y Deportes, 126(4), 37-45.

Villalón, M. (2016). Alfabetización Inicial: Claves de acceso al aprendizaje de la lectura y la escritura desde los primeros meses de vida (2.a ed.). Ediciones Universidad Católica.

Yangin Eksi, G., \& Gungor, M. N. (2018). Exploring the Use of Narratives to Understand Pre-service Teachers' Prácticum Experiences from a Sociocultural Perspective. Australian Journal of Teacher Education, 43(4), 159-174. 